\title{
401.
}

\section{A NOTATION OF THE POINTS AND LINES IN PASCAL'S THEOREM.}

[From the Quarterly Journal of Pure and Applied Mathematics, vol. Ix. (1868), pp. 268-274.]

TAKING six points $1,2,3,4,5,6$ on a conic; let $A, B, C, D, E, F, G, H, I, J, K, L, M, N, O$ denote each a combination of three lines, thus

$$
\left|\begin{array}{l|l|l}
12.34 .56=A & 12.35 .64=F & 12.36 .45=K \\
13.45 .62=B & 13.46 .25=G & 13.42 .56=L \\
14.56 .23=C & 14.52 .36=H & 14.53 .62=M \\
15.62 .34=D & 15.63 .42=I & 15.64 .23=N \\
16.23 .45=E & 16.24 .53=J & 16.25 .34=O
\end{array}\right|
$$

then any hexagon formed with the six points may be represented by a combination of some two of the letters $A, B, \& c$., viz. the three alternate sides are the lines represented by one letter, and the other three alternate sides the lines represented by the other letter: for example, the hexagon 123456 is $A E$; and so for the other hexagons. Any duad $A E$ thus representing a hexagon may be termed a hexagonal duad; the number of such duads is sixty. Each Pascalian line may be denoted by the symbol of the hexagon to which it belongs; thus, the line which belongs to the hexagon $A E$, is the line $A E$.

I form the following combinations:

$I M O$. DHJ each involving all the duads 12 , \&c. except those of 123.456, $D E G . B N O$

$E L M . B C J$

$\begin{array}{ll}" & " \\ " & " \\ " & " \\ " & " \\ " & "\end{array}$

124. 356 ,

$H L N . C G I$

125 . 346 ,

EFI.JKN

AEH . CKO

$A M N . C D F$

AGJ. ELO

$A B I$. DKL

GKM.BFH 
and also the combinations:

$A E G M I$ involving all the duads 12,13 , \&c.,

$\begin{array}{lll}A B H J N & " & " \\ B C F I O & " & " \\ C D G J K & , & " \\ D E F H L & , & " \\ K L M N O & , & ,\end{array}$

which I call respectively the ten-partite and six-partite arrangements. It is to be remarked that (considering $I M O . D H J$ as standing for the six duads $I M, I O, M O$ $D H, D J, H J$, and so for the others) the ten-partite arrangement contains all the sixty hexagonal duads: and in like manner, (considering $A E G M I$ as standing for the ten duads $A E, A G, A M, A I, E G, E M, E I, G M, G I, M I$, and so for the others) the six-partite arrangement contains all the sixty hexagonal duads.

The 60 Pascalian lines intersect by 4's in the 45 Pascalian points $p$, by 3 's in 20 points $g$ and in 60 points $h$, and by 2's in 90 points $m, 360$ points $r$, 360 points $t$, 360 points $z$, and 9 points $w$.

The intersections of the Pascalian lines thus are

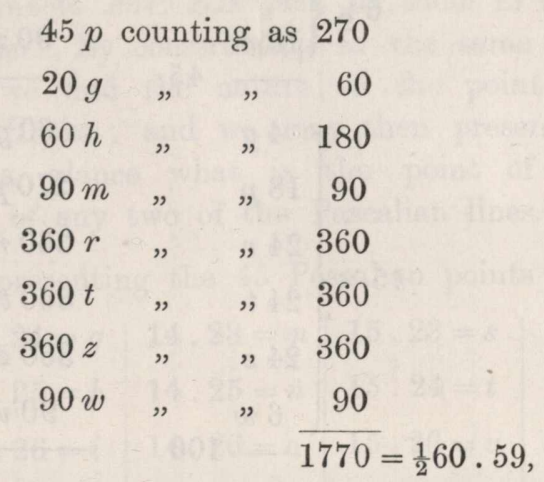

and the intersections on each Pascalian line are

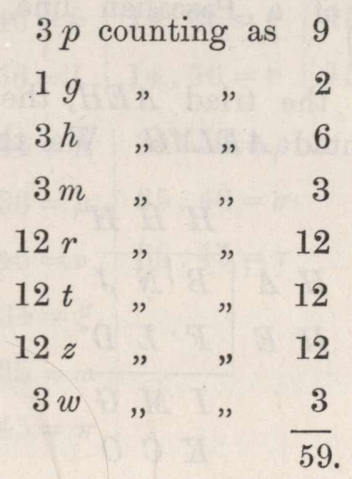


For the ten-partite arrangement, any double triad such as $A B I . D K L$ gives 15 intersections; $10 \times 15=150$; and any pair of double triads such as $A B I . D K L$ and $A E H . C K O$ gives 36 intersections; $45 \times 36=1620$; and these are

$$
\begin{array}{r}
10 \times \begin{cases}6 g & 60 g \\
9 m & 90 m\end{cases} \\
\hline 45 \times \begin{cases}6 p & 270 p \\
4 h & 180 h \\
8 r & 360 r \\
8 t & 360 t \\
8 z & 360 z \\
2 w & 90 w \\
\frac{1620}{1770}\end{cases}
\end{array}
$$

For the six-partite arrangement any pentad such as $A B H J N$ gives 45 intersections; $6 \times 45=270$; and any two pentads such as $A B H J N$ and $A E G M I$ give 100 intersections; $15 \times 100=1500$; and these are

$$
\begin{aligned}
& 6 \times\left\{\begin{array}{ll}
30 h & 180 h \\
15 m & 90 m \\
\hline & 45
\end{array} 270\right. \\
& 15 \times \begin{cases}4 g & 60 g \\
18 p & 270 p \\
24 r & 360 r \\
24 t & 360 t \\
24 z & 360 z \\
6 w & 90 w \\
\hline & \frac{1500}{1770}\end{cases}
\end{aligned}
$$

I analyse the intersections of a Pascalian line, say $A E$, by the remaining 59 Pascalian lines as follows:

Observe that $A E$ belongs to the triad $A E H$, the complementary triad whereof is $C K O$; it also belongs to the pentad AEIMG. We thus obtain, corresponding to $A E$, the arrangement

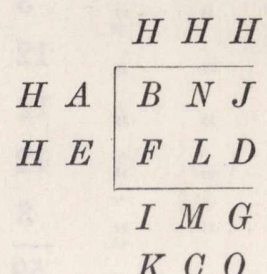


viz. $H A B N J$, is the pentad which contains $H A$, the arrangement of the last three letters $B, N, J$ thereof being arbitrary; $H E F L D$ is the pentad that contains $H E$, but the last three letters are so arranged that the columns $H B F, H N L, H J D$ are each of them a triad, $I M G$ is then the residue of the pentad $A E I M G$, and $K C O$ is the complementary triad to $A E H$, but the arrangement of the letters $I M G$, and of the letters $K C O$, are each of them determinate; viz. these are such that we have $B F I C O$, NLMKO, JDGCK, each of them a pentad.

And this being so we derive from the arrangement

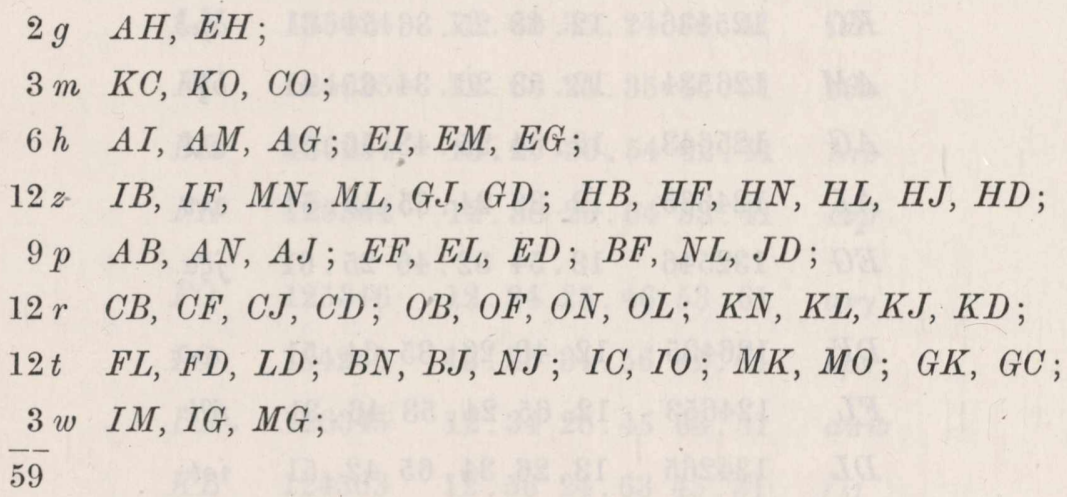

viz. the line $A E$ in question meets $A H, E H$ each of them in a point $g ; K C, K O, C O$ each in a point $m$; and so on. By constructing in the same way an arrangement for each of the lines $A H$, \&c., we find the nature of the point of intersection of any two of the lines $A B, A E, A H, \& c$; and we may then present the results in a table (see Plate), which shows at a glance what is the point of intersection (whether a point $g, m, h, z, p, r, t$, or $w)$ of any two of the Pascalian lines.

I further remark that representing the 45 Pascalian points as follows:

\begin{tabular}{l|l|l|l|l}
$12.34=a$ & $13.24=g$ & $14.23=m$ & $15.23=s$ & $16.23=y$ \\
$12.35=b$ & $13.25=h$ & $14.25=n$ & $15.24=t$ & $16.24=z$ \\
$12.36=c$ & $13.26=i$ & $14.26=0$ & $15.26=u$ & $16.25=\alpha$ \\
$12.45=d$ & $13.45=j$ & $14.35=p$ & $15.34=v$ & $16.34=\beta$ \\
$12.46=e$ & $13.46=k$ & $14.36=q$ & $15.36=w$ & $16.35=\gamma$ \\
$12.56=f$ & $13.56=l$ & $14.56=r$ & $15.46=x$ & $16.45=\delta$ \\
$23.45=\epsilon$ & $25.34=\lambda$ & $34.56=\rho$ \\
$23.46=\zeta$ & $25.36=\mu$ & $35.46=\sigma$ \\
$23.56=\eta$ & $25.46=\nu$ & $36.45=\tau$ \\
$24.35=\theta$ & $26.34=\xi$ & \\
$24.36=\iota$ & $26.35=\omega$ & \\
$24.56=\kappa$ & $26.45=\pi$ &
\end{tabular}


A NOTATION OF THE POINTS AND LINES IN PASCAL'S THEOREM. [401 the sixty hexagons and their Pascalian lines then are

$\begin{array}{llllll}A E & 123456 & 12.45 & 23.56 & 34.61 & d \eta \beta \\ A H & 125634 & 12.63 & 25.34 & 56.41 & c \lambda r \\ E H & 145236 & 14.23 & 45.36 & 52.61 & m \tau \alpha \\ C K & 123654 & 12.65 & 23.54 & 36.41 & f \epsilon q \\ C O & 143256 & 14.25 & 43.56 & 32.61 & n \rho y \\ K O & 125436 & 12.43 & 25.36 & 54.61 & a \mu \delta \\ A M & 126534 & 12.53 & 26.34 & 65.41 & b \xi r \\ A G & 125643 & 12.64 & 25.43 & 56.31 & e \lambda l \\ A I & 124365 & 12.36 & 24.65 & 43.51 & c \kappa v \\ E G & 132546 & 13.54 & 32.46 & 25.61 & j \zeta \alpha \\ D F & 126435 & 12.43 & 26.35 & 64.51 & a \omega x \\ F L & 124653 & 12.65 & 24.53 & 46.31 & f \theta k \\ D L & 134265 & 13.26 & 34.65 & 42.51 & i \rho t \\ B N & 132645 & 13.64 & 32.45 & 26.51 & k \epsilon u \\ B J & 135426 & 13.42 & 35.26 & 54.61 & g \omega \delta \\ J N & 153246 & 15.24 & 53.46 & 32.61 & t \sigma y \\ G K & 125463 & 12.46 & 25.63 & 54.31 & e \mu j \\ K M & 126354 & 12.35 & 26.54 & 63.41 & b \pi q \\ I O & 152436 & 15.43 & 52.36 & 24.61 & v \mu z \\ M O & 143526 & 14.52 & 43.26 & 35.61 & n \xi \gamma \\ E M & 145326 & 14.32 & 45.26 & 53.61 & m \pi \gamma \\ E I & 154236 & 15.23 & 54.36 & 42.61 & s \tau z \\ A N & 123465 & 12.46 & 23.65 & 34.51 & e \eta v \\ A J & 124356 & 12.35 & 24.56 & 43.61 & b \kappa \beta \\ A B & 126543 & 12.54 & 26.43 & 65.31 & d \zeta l \\ D E & 154326 & 15.32 & 54.26 & 43.61 & s \pi \beta \\ E L & 132456 & 13.45 & 32.56 & 24.61 & j \eta z \\ E F & 123546 & 12.54 & 23.46 & 35.61 & d \zeta \gamma \\ C D & 143265 & 14.26 & 43.65 & 32.51 & o \rho s \\ C F & 123564 & 12.56 & 23.64 & 35.41 & f \zeta p\end{array}$


A NOTATION OF THE POINTS AND LINES IN PASCAL'S THEOREM.

$\begin{array}{lllllll}C G & 132564 & 13.56 & 32.64 & 25.41 & l \zeta n\end{array}$

$\begin{array}{llllll}C I & 142365 & 14.36 & 42.65 & 23.51 & q \kappa s\end{array}$

MN $146235 \quad 14.2346 .3562 .51 \quad$ mби

$\begin{array}{llllll}G J & 135246 & 13.24 & 35.46 & 52.61 & g \sigma \alpha\end{array}$

$B I \quad 13624 \check{5} 13.2436 .4562 .51 \quad g \tau u$

DG $\quad 134625 \quad 13.6234 .25 \quad 46.51 \quad i \lambda x$

$L M \quad 135624 \quad 13.6235 .2456 .41 \quad i \theta r$

$\begin{array}{llllll}F I & 124635 & 12.63 & 24.35 & 46.51 & c \theta x\end{array}$

BH 136254， $13.2536 .54 \quad 62.41$ hro

$\begin{array}{llllll}F H & 125364 & 12.36 & 25.64 & 53.41 & c \nu p\end{array}$

FO $\quad 125346 \quad 12.34 \quad 25.46 \quad 53.61 \quad$ avy

LO $\quad 134256 \quad 13.25 \quad 34.56 \quad 42.61 \quad h \rho z$

DK $\quad 126345 \quad 12.3426 .4563 .51 \quad a \pi w$

$\begin{array}{llllll}K L & 124563 & 12.56 & 24.63 & 45.31 & f_{i j}\end{array}$

$\begin{array}{llllll}\text { BO } & 134526 & 13.52 & 34.26 & 45.61 & h \xi \delta\end{array}$

NO $152346 \quad 15.3452 .4623 .61$ vvy

$\begin{array}{llllllll}B C & 132654 & 13.65 & 32.54 & 26.41 & l \epsilon o\end{array}$

$\begin{array}{llllll}C J & 142356 & 14.35 & 42.56 & 23.61 & \text { рку }\end{array}$

$\begin{array}{llllll}J K & 124536 & 12.53 & 24.36 & 45.61 & b \iota \delta\end{array}$

KN $123645 \quad 12.6423 .4536 .51$ eєw

DH $\quad 143625 \quad 14.6243 .2536 .51$ odw

HJ $\quad 142536 \quad 14.5342 .3625 .61 \quad p \iota \alpha$

$H L \quad 136524 \quad 13.5236 .2465 .41$ hır

$H N \quad 146325 \quad 14.3246 .2563 .51 \quad m \nu w$

$\begin{array}{llllll}B F & 126453 & 12.46 & 26.53 & 64.31 & d \omega k\end{array}$

DJ $\quad 153426 \quad 15.4253 .26 \quad 34.61 \quad t \omega \beta$

$\begin{array}{llllll}L N & 132465 & 13.46 & 32.65 & 24.51 & k \eta t\end{array}$

GM $\quad 135264 \quad 13.2635 .6452 .41 \quad i \sigma n$

IM $142635 \quad 14.63 \quad 42.3526 .51 \quad q \theta u$

GI $136425 \quad 13.4236 .2564 .51 \quad g \mu x$

c. VI. 
Each Pascalian point belongs to four different hexagons; viz. $a$ to the hexagons $K D, K O, F D, F O$; and so for the other points, thus:

$$
\begin{array}{llll}
a & (K, F)(D, O) & x & (D, I)(F, G) \\
b & (A, K)(M, J) & y & (C, N)(J, O) \\
c & (A, F)(H, I) & z & (E, O)(I, L) \\
d & (A, F)(B, E) & \alpha & (E, J)(G, H) \\
e & (A, K)(G, N) & \beta & (A, D)(E, J) \\
f & (C, L)(K, F) & \gamma & (E, O)(F, M) \\
g & (B, G)(I, J) & \delta & (B, K)(J, O) \\
h & (B, L)(H, O) & \epsilon & (B, K)(C, N) \\
i & (D, M)(G, L) & \zeta & (C, E)(F, G) \\
j & (E, K)(G, L) & \eta & (A, L)(E, N) \\
k & (B, L)(F, N) & \theta & (F, M)(I, L) \\
l & (A, C)(B, G) & \boldsymbol{1} & (H, K)(J, L) \\
m & (E, N)(H, M) & \kappa & (A, C)(I, J) \\
n & (C, M)(G, O) & \lambda & (A, D)(G, H) \\
o & (B, D)(C, H) & \mu & (G, O)(I, K) \\
p & (C, H)(F, J) & \nu & (F, N)(H, O) \\
q & (C, M)(I, K) & \xi & (A, O)(B, M) \\
r & (A, L)(H, M) & \omega & (B, D)(F, J) \\
s & (C, E)(D, I) & \pi & (D, M)(E, K) \\
t & (J, L)(D, N) & \rho & (C, L)(D, O) \\
u & (B, M)(I, N) & \sigma & (G, N)(J, M) \\
v & (A, O)(N, I) & \tau & (B, E)(H, I) \\
w & (D, N)(H, K) & &
\end{array}
$$

I have constructed on a very large scale a figure of the sixty Pascalian lines, and the forty-five Pascalian points, marking them according to the foregoing notation; but the figure is from its complexity, and the inconvenient way in which the points are either crowded together or fly off to a great distance, almost unintelligible. 